\title{
BIPACKINGS OF PAIRS INTO TRIPLES, AND ISOMORPHISM CLASSES OF SMALL BIPACKINGS
}

\author{
R. G. STANTON, M. J. ROGERS, R. F. QUINN and D. D. COWAN
}

(Received 29 March 1982)

Communicated by W. D. Wallis

\begin{abstract}
The $(2,3, v)$ bipacking number is determined for all integers $v$, and the number of non-isomorphic bipackings is found for small values of $v$. The general solution for lambda packings of pairs into triples is deduced from the results for $\lambda=1$ and $\lambda=2$.
\end{abstract}

1980 Mathematics subject classification (Amer. Math. Soc.): 05 B 40)

\section{Introduction}

The packing number $D(t, k, v)$ is defined as the maximal cardinality of a family of $k$-sets, selected from a $v$-set, such that no $t$-set is repeated in the family. To avoid trivial cases, we normally require $t<k<v$.

The packing number $D(t, k, v)$ can be generalized by allowing the family of $k$-sets to have any $t$-set repeated up to a maximum of $\lambda$ times. We then write $D_{\lambda}(t, k, v)$ for the cardinality of the family; thus $D_{1}(t, k, v)=D(t, k, v)$. If $\lambda=2$, we denote the packing by the special name of bipacking. As in the case of packings, we define a bipacking to be perfect if every $t$-set occurs exactly twice.

The first results on packings were for $t=2, k=3$; an account can be found in [8]. We intend to describe the analogous situation for $t=2, k=3, \lambda=2$, that is, for bipackings of pairs into triples. First, we require

LEMMA $1 . D_{2}(1,2, v)=v$.

Copyright Australian Mathematical Society 1983 
Proof. If $v=2 a$, then one can take $a$ pairs which exactly cover the $2 a$ elements once each. A second selection of $a$ pairs, which may be identical with the first, can then be adjoined to give a total of $2 a$ pairs. Since every element occurs twice, the bipacking is perfect and can not be extended.

If $v=2 a+1$, select $a$ pairs containing all elements except $x$. Then take another selection of $a$ pairs containing all elements except $y$, where $y \neq x$. These $2 a$ pairs, together with $x y$, give a bipacking with $2 a+1$ pairs.

We can now give the lower bound for $D_{2}(2,3, v)$; the method is well-known (see [8], for example). If we assume a general bipacking with $D_{2}(t, k, v)$ blocks, then the bipacking array contains $k D_{2}(t, k, v)$ elements. However, the frequency of a specific element $x$ can never exceed $D_{2}(t-1, k-1, v-1)$, and there are $v$ such elements. So the array contains a maximum of $v D_{2}(t-1, k-1, v-1)$ elements. Thus, we have

LEMMA 2. $D_{2}(t, k, v) \leqslant\left\lfloor\frac{t}{k} D_{2}(t-1, k-1, v-1)\right\rfloor$.

In our particular case, we may set $t=2, k=3$, and use the results of the preceding lemma to give

Lemma 3. $D_{2}(2,3, v) \leqslant\lfloor v(v-1) / 3\rfloor$.

Now, if $v \equiv 0$ or 1 (modulo 3), it is well known that there exists a Balanced Incomplete Block Design with parameters $(v, v(v-1) / 3, v-1,3,2)$. (For some particularly nice constructions of these designs, see [9].) So we may state

THEOREM 1. If $v \equiv 0$ or 1 (modulo 3), then there exists a perfect bipacking of pairs into triples, and the number of such bipackings is the number of non-isomorphic $B I B D s$ with parameters $(v, v(v-1) / 3, v-1,3,2)$.

\section{The bipackings for $v=6 a+2$}

If $v=6 a+2$, then the bound is $\lfloor(6 a+2)(6 a+1) / 3\rfloor=12 a^{2}+6 a$. The total number of triples would contain $36 a^{2}+18 a$ elements and the same number of pairs. But the total number of pairs from $6 a+2$ elements is $(6 a+2)(6 a+1)$, since each pair must occur twice, and this is $36 a^{2}+18 a+2$. Now the maximum frequency of any element is $6 a+1$ and, if the frequency drops to $6 a$, then two 
pairs are missing. Hence, we have

THEOREM 2. If $v=6 a+2$, then a bipacking with $12 a^{2}+6 a$ blocks can exist only if all elements occur with frequency $6 a+1$, with the exception of two specific singular elements $A$ and $B$; these two elements have frequencies $6 a$, and all pairs occur twice except for the pair $A B$, which does not occur at all.

We note that, if $x_{i}$ denote the intersection numbers of a base block $B_{1}$ with other blocks, where $B_{1}$ contains neither $A$ nor $B$, then

$$
\begin{gathered}
x_{0}+x_{1}+x_{2}+x_{3}=12 a^{2}+6 a-1, \\
x_{1}+2 x_{2}+3 x_{3}=3(6 a), \\
x_{2}+3 x_{3}=3 .
\end{gathered}
$$

Hence we obtain

THEOREM 3. If $B_{1}$ contains only elements of frequencies $6 a+1$, then its intersection numbers are either $x_{0}=12 a^{2}-12 a+1, x_{1}=18 a-3, x_{2}=0, x_{3}=1$; or they are $x_{0}=12 a^{2}-12 a+2, x_{1}=18 a-6, x_{2}=3, x_{3}=0$.

We use this result to deduce all bipackings on eight elements. The bound is 18 , and, if it is attained, there are six blocks containing $A$, six containing $B$, and six containing only $1, \ldots, 6$. If we assume that block 123 is repeated, then we get blocks

$123, \quad 123, \quad 145, \quad 246,356,456$.

The first blocks are then

$$
\begin{array}{llllll}
A 1 X, & A 1 X, & A 2 X, & A 2 X, & A 3 X, & A 3 X, \\
B 1 X, & B 1 X, & B 2 X, & B 2 X, & B 3 X, & B 3 X,
\end{array}
$$

and we need pairs $14,15,16,16,24,25,25,26,34,34,35,36$. If we assume that there are no further repeated blocks, then we can write

$$
A 16, B 16, A 25, B 25, A 34, B 34 \text {, }
$$

and complete the design uniquely with

$A 14, B 15, \quad B 24, \quad A 26, \quad A 35, B 36$.

On the other hand, if a pair is repeated we may write $A 16, A 16, B 14, B 15, B 26, B 36$.

This forces us to select $A 25, A 34$ and complete the design with either $A 25, A 34$, $B 24, B 35$, or with $A 24, A 35, B 25, B 34$. We thus have 
THEOREM 4. There are three non-isomorphic bipackings on eight elements which contain repeated blocks on the non-singular elements, and these contain a total or one, two, or four repeated blocks. The bipackings can be written as

$$
123,123,145,246,356,456 \text {, }
$$

together with

(A)

or

or

(C)
$A 16, A 25, \quad A 34, A 14, A 26, A 35$, $B 16, \quad B 25, B 34, \quad B 15, \quad B 24, \quad B 36$;

$A 16, A 16, A 25, A 25, A 34, A 34$, $B 14, B 15, B 26, B 36, B 24, B 35$;

$A 16, A 16, A 25, A 24, A 34, A 35$, $B 14, B 15, B 26, B 36, B 25, B 34$.

Now let us assume that there are no repeated blocks on $1, \ldots, 6$, and take a block 123. If there is a block 456, then the other four blocks in the non-singular set must contain two each of $1, \ldots, 6$. Since there are two blocks disjoint to 123 (and to 456), there must be exactly one pair of the form $A 23$ (and one pair of the form $A 56$ ) in the first 12 blocks. So we may take the last six blocks as including

$$
12 X, 13 X, 2 X X, 3 X X,
$$

where the $X$ 's denote elements from $\{4,5,6\}$. There is no loss in generality in writing

$$
12 X, \quad 13 X, 245, \quad 346,
$$

and thus we get
(A)
$125, \quad 136, \quad 245, \quad 346$,

or

$$
126, \quad 135, \quad 245,346 .
$$

In case $(\mathrm{A})$, we need blocks

$$
\begin{aligned}
& A 1 X, \quad A 1 X, \quad A 2 X, \quad A 3 X, \quad A 23, \quad A 56, \\
& B 1 X, \quad B 1 X, \quad B 2 X, \quad B 2 X, \quad B 3 X, \quad B 3 X,
\end{aligned}
$$

and we need pairs $14,14,15,16,24,26,26,34,35,35$.

If there are no repeated blocks, we take $A 14, B 14, A 26, B 26, A 35, B 35$, and can not complete $A 1 X$. So we must take a repeated block. If 14 is repeated, we get
$A 14, A 14, A 26, A 35, A 23, A 56$,
$B 15, \quad B 16, \quad B 24, \quad B 26, \quad B 34, \quad B 35$; 
or we get

$A 15, A 16, A 24, A 34, A 23, A 56$, $B 14, B 14, B 26, B 26, B 35, \quad B 35$.

If 14 is not repeated, but 26 is, we get (the case of a repeated 35 is isomorphic via the permutation (23)(56))

$A 14, B 14, B 26, B 26, A 24, A 35$, $A 16, B 15, B 34, B 35$.

In case (B), we need blocks

$A 1 X, \quad A 1 X, \quad A 2 X, \quad A 3 X, \quad A 23, \quad A 56$, $B 1 X, \quad B 1 X, \quad B 2 X, \quad B 2 X, \quad B 3 X, \quad B 3 X$,

and we need pairs $14,14,15,16,24,25,26,34,35,36$. We are thus forced to select

(B1) $A 14, A 14, A 25, A 36, \quad B 15, \quad B 16, \quad B 24, \quad B 26, \quad B 34, \quad B 35$;

or

(B2) $B 14, B 14, A 15, A 16, A 24, A 34, B 25, B 26, B 35, B 36$;

or

(B3) $A 14, B 14, A 15, B 16, A 24, A 36, B 25, B 26, B 34, B 35$;

or

(B4) $A 14, B 14, A 15, B 16, A 26, A 34, B 24, B 25, B 35, B 36$.

Thus we have

THEOREM 5. If the non-singular blocks of a $(2,3,8)$ bipacking contain two disjoint unrepeated blocks, then there are seven such non-isomorphic bipackings.

Finally, let us assume that the last six blocks contain 123 but not 456 . We then have five blocks containing symbols $1,1,2,2,3,3,4,4,4,5,5,5,6,6,6$. If there are $x$ blocks of the form $a a b$ and $y$ blocks of the form $a b b$, where $a \in\{1,2,3\}$ and $b \in\{4,5,6\}$, we find that $x=1, y=4$, and hence we must take the blocks as

$$
X 45, X 45, X 46, X 56, X X 6 \text {, }
$$

where $X \in\{1,2,3\}$.

We may then assign the blocks to be $123, \quad 145, \quad 245, \quad 346, \quad 126,356 ;$

or

(D) $\quad 123,145,245,346,136,256$. 
Case (C) leads to

$A 1 X, \quad A 13, \quad A 23, \quad A 2 X, \quad A 46, \quad A 56$, $B 1 X, \quad B 1 X, \quad B 2 X, \quad B 2 X, \quad B 3 X, \quad B 3 X$;

or $A 1 X, \quad A 13, \quad A 2 X, A 2 X, \quad A 3 X, A X X$, $B 1 X, B 1 X, B 2 X, B 23, \quad B 3 X, B X X$.

$\left(C_{1}\right)$ requires pairs $14.15,16,24,25.26,34,35$, and hence completes to $A 13, \quad A 23, \quad A 46, \quad A 56, \quad B 16, \quad B 26, \quad B 34, B 35$, $A 14, \quad A 25, \quad B 15, \quad B 24$

(use (45) to prescribe $A 14$ ).

$\left(\mathrm{C}_{2}\right)$ also requires pairs $14,15,16,24,25,26,34,35,46,56$, and can be written (by use of the permutation (45)) as $A 1 X, \quad A 13, \quad A 2 X, \quad A 2 X, \quad A 3 X, \quad A 46$, $B 1 X, \quad B 1 X, \quad B 2 X, \quad B 23, \quad B 3 X, \quad B 56$.

This case completes to

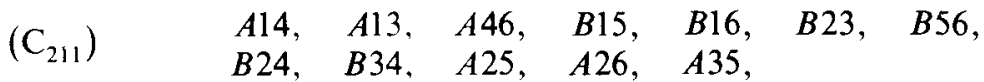

or

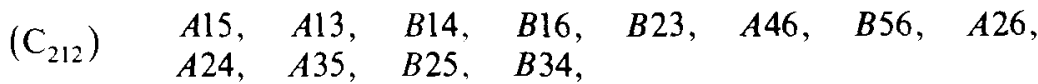

or

$\left(\mathrm{C}_{213}\right)$

$\mathrm{C}_{212}$ with the last four blocks as $A 25, A 34, B 35, B 24$,

or

$\left(\mathrm{C}_{214}\right) \quad A 16, \quad A 13, \quad A 46, \quad B 14, \quad B 15, \quad B 23, \quad B 56$, $B 26, B 34, B 35, A 24, A 25$.

In case (D), with blocks

$123,145,245,136,346,256$,

we need pairs $14,15,16,24,26,34,35,35$, in the skeleton $A 1 X, \quad A 12, \quad A 23, \quad A 3 X, \quad A 46, \quad A 56$, $B 1 X, \quad B 1 X, \quad B 2 X, \quad B 2 X, \quad B 3 X, \quad B 3 X$,

or $A 1 X, \quad A 12, \quad A 2 X, \quad A 3 X, \quad A 3 X, \quad A X X$, $B 1 X, \quad B 1 X, \quad B 2 X, \quad B 23, \quad B 3 X, \quad B X X$.

(D $)$ can be completed by $A 14, A 35$, or $A 15, A 34$ (2 ways). ( $\mathrm{D}_{2}$ ) can be written as: $A 1 X, \quad A 12, \quad A 2 X, \quad A 3 X, \quad A 3 X, \quad A 46$, $B 1 X, \quad B 1 X, \quad B 2 X, \quad B 23, \quad B 3 X, B 56$, 
or

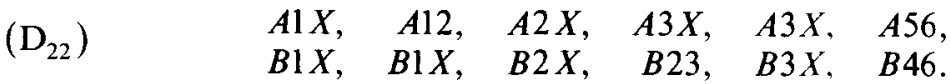

$\left(\mathrm{D}_{21}\right)$ can be completed using $A 35, A 35, A 24, A 16$, or by using $A 35, A 35, A 14$, $A 26$; or by using $A 35, A 34, A 15, A 26 .\left(\mathrm{D}_{22}\right)$ can be completed using $A 34, A 35$, $A 14, A 26$, or by using $A 34, A 35, A 16, A 24$. These results can be summed up in

THEOREM 6. In case the non-singular blocks of a $(2,3,8)$ bipacking do not contain two disjoint blocks, then there are twelve non-isomorphic bipackings.

Combination of the last three theorems finally gives

THEOREM 7. There are twenty-two non-isomorphic $(2,3,8)$ bipackings.

\section{The bipacking number for $v=6 a+2$}

In the last section, we obtained the upper bound for $D_{2}(2,3,6 a+2)$ as $12 a^{2}+6 a$. We now show that this bound is always attained.

First, we refer to Theorem 14.3 of [7] and modify it slightly. In that theorem, four results were shown; these will be summarized here.

(a) Triples can be written on the integers $0 \leqslant x \leqslant 12 k$ such that all differences from 1 to $12 k$ occur and the differences in a triple are such that one difference is the sum of the other two. For example, if $k=1$, then the triples $(0,1,6),(0,2,11)$, $(0,3,10),(0,4,12)$, give rise to differences such that $1+5=6,2+9=11$, $3+7=10,4+8=12$.

(b) Triples can be written on the integers $0 \leqslant x \leqslant 12 k+3$ such that all differences from 1 to $12 k+3$ occur and the differences in a triple are such that one difference is the sum of the other two. For example, if $k=1$, then triples $(0,1,7),(0,2,14),(0,3,11),(0,4,13),(0,5,15)$, give rise to differences such that $1+6=7,2+12=14,3+8=11,4+9=13,5+10=15$.

(c) Triples can be written on the integers $0 \leqslant x \leqslant 12 k+7$ such that all differences from 1 to $12 k+7$ occur with the exception of $12 k+6$; furthermore, the differences in a triple are such that one difference is the sum of the other two, and there is a triple whose differences are $2,12 k+5,12 k+7$. For example, if $k=1$, then triples $(0,1,9),(0,2,19),(0,3,15),(0,4,14),(0,5,16),(0,6,13)$, give rise to differences such that $1+8=9,2+17=19,3+12=15,4+10=14$, $5+11=16,6+7=13$.

(d) Triples can be written on the integers $0 \leqslant x \leqslant 12 k+10$ such that all differences from 1 to $12 k+10$ occur with the exception of $12 k+9$; furthermore, 
the differences in a triple are such that one difference is the sum of the other two, and there is a triple with differences $2,12 k+8,12 k+10$. For example, if $k=1$, then triples $(0,1,19),(0,2,22),(0,3,13),(0,4,16),(0,5,14),(0,6,17),(0,7,15)$, give rise to differences such that $1+18=19,2+20=22,3+10=13,4+12$ $=16,5+9=14,6+11=17,7+8=15$.

We now modify this theorem to give the following result.

THEOREM 8. Let an integer $s=12 k+u$ be given $(u=0,3,6,9)$. Then it is possible to divide the integers in the interval $0 \leqslant x \leqslant 12 k+u$ into triples, not necessarily all distinct, such that these triples, together with two pairs, will generate all differences modulo $24 k+2 u$ exactly twice.

Proof. The general proof can be most easily illustrated by looking at the four cases and doing the constructions in the case $k=1$.

(a) First we consider $s=12 k$, and refer to the triples guaranteed by Theorem 14.3 of [7]. For example, if $s=12$, they are $(0,1,6),(0,2,11),(0,3,10),(0,4,12)$. Duplicate these triples except for the triple containing 12 . Keep only one copy of it, and use pairs $(0,4)$ and $(0,8)$. Each triple now generates six differences (modulo $24 k$ ), and the fact that $12 k \equiv-12 k$ explains the omission of the repeat on the difference $12 k$.

(b) Now we consider $s=12 k+3$. Proceed as in part (a), modulo $24 k+6$. For example, if $k=1$, we get triples $(0,1,7),(0,2,14),(0,3,11),(0,4,13)$, all repeated twice, along with $(0,5,15),(0,5),(0,10)$.

(c) In this case, $s=12 k+6$, and we work modulo $24 k+12$. Here we use the triples involving numbers up to $12 k+4$ only, that is, we delete the triple involving differences $2,12 k+5,12 k+7$. When we duplicate these triples, we have all differences modulo $24 k+12$ occurring twice except for $2,2,12 k+5$, $12 k+5,12 k+6$. To get these, we take the triple $(0,2,12 k+7)$ and the pairs $(0,2),(0,12 k+6)$, noting that $12 k+7 \equiv-(12 k+5)$.

(d) In this case, $s=12 k+9$, and we work modulo $24 k+18$. The construction uses the previous triples from (d), and proceeds exactly as in part (c). Thus, all triples are duplicated save that involving the differences $2,12 k+8,12 k+10$; it is replaced by $(0,2,12 k+10)$ and the pairs $(0,2),(0,12 k+9)$.

The theorem just proved allows us to construct cyclically generated bipackings for $v=6 a+2$. We consider the two singular elements of frequency $6 a$ to act as infinite elements and we cycle the other $6 a$ elements (of frequency $6 a+1$ ) modulo $6 a$. There are altogether $6 a$ blocks containing $A, 6 a$ containing $B$, and $12 a^{2}-6 a$ containing only $1,2, \ldots, 6 a$. Divide the situation into four cases according as $6 a$ takes the form $24 k, 24 k+6,24 k+12,24 k+18$. By Theorem 
8, we always get two pairs and a collection of triples such that all differences modulo $6 a$ occur exactly twice. Put $A$ with one pair, $B$ with the other pair, and keep the triples; then develop these triples cyclically modulo $6 a, A$ and $B$ acting as infinities. This constructs our required bipacking in $6 a(2 a-1)$ blocks. Hence, we have

Theorem 9. $D_{2}(2,3,6 a+2)=12 a^{2}+6 a$.

We illustrate the procedure by giving the bipackings constructed by this algorithm for $v=8,14,20,26$.

EXAmple 1. Elements $1,2,3,4,5,6, A, B$. This is case (b), and we use the triple $(0,1,3)$ and the pairs $(0,1),(0,2)$. Hence we write the bipacking as $(1,3,6)$, $(A, 1,6),(B, 2,6)$, modulo 6 , where we write 6 rather than 0 . In developed form, it can be depicted as

$\begin{array}{lll}136 & A 16 & B 26 \\ 241 & A 21 & B 31 \\ 352 & A 32 & B 42 \\ 463 & A 43 & B 53 \\ 514 & A 54 & B 64 \\ 625 & A 65 & B 15\end{array}$

A more interesting case occurs for $v=32$; the theorem then generates the bipacking from the blocks
$(1,7,30)$,
$(1,7,30)$
$(2,14,30)$,
$(2,14,30)$,
$(3,11,30)$,
$(3,11,30)$,
$(4,13,30)$,
$(4,13,30)$,
$(5,15,30)$,
$(A, 5,30)$,
$(B, 10,30)$, modulo 30 .

EXAmple 2. If $v=14$, we have $A, B$, and 12 other elements; so we use case (c). The theorem gives a bipacking generated by

$$
(1,4,12), \quad(1,4,12), \quad(2,7,12), \quad(A, 2,12), \quad(B, 2,12), \text { modulo } 12 .
$$

EXAMPLE 3. If $v=20$, we have $A, B$, and 18 other elements; so we use case (d). The theorem gives a bipacking generated by $(1,6,18),(1,6,18),(3,7,18),(3,7,18)$, $(2,10,18),(A, 2,18),(B, 9,18)$, modulo 18 .

EXAMPLE 4. If $v=26$, we have $A, B$, and 24 other elements; so we use case (a). The theorem gives a bipacking generated by $(1,6,24),(1,6,24),(2,11,24)$, $(2,11,24),(3,10,24),(3,10,24),(4,12,24),(A, 4,24),(B, 8,24)$, modulo 24 . 


\section{Bipacking number for $v=6 a+5$}

There are certain similarities between the cases $v=6 a+5$ and $v=6 a+2$. First, we easily find that the bound is $12 a^{2}+18 a+6$, and this is achieved if and only if there are two singular elements $A$ and $B$ of frequency $6 a+3$ each and $6 a+3$ elements, each with frequency $6 a+4$. The construction given to achieve the bound is as follows.

Take a resolvable triple system (this is always possible; see [10]) on the $6 a+3$ elements and duplicate it to give a resolvable $\mathrm{BIBD}$ with parameters

$$
(6 a+3,(6 a+2)(2 a+1), 6 a+2,3,2) .
$$

This design will have $6 a+2$ resolution classes. Take all the triples in $6 a$ of these classes, and let the remaining resolutions be called $R(A)$ and $R(B)$. Split the triples of $R(A)$ into pairs, three from each triple, thus giving a total of $6 a+3$ pairs; associate these pairs with symbol $A$. In the same way, use $R(B)$ to produce $6 a+3$ pairs to associate with symbol $B$. This produces a bipacking for $v=6 a+5$ as described in

THEOREM 10. $D_{2}(2,3,6 a+5)=12 a^{2}+18 a+6$, and the bipacking has two singular elements $A$ and $B$ of frequency $6 a+3$. The pair $A B$ does not occur, but all other pairs occur twice, and all elements other than $A$ and $B$ have frequency $6 a+4$.

EXAMPLE 5. Let $v=11$, and take the affine plane on $1, \ldots, 9$, in the usual form $123,456,789 ; 147,258,369 ; 159,267,348 ; 168,249,357$. Then we get our bipacking of 36 triples as follows.

Take the 12 triples of the affine plane and then repeat all of them except the six triples of the last two resolutions; this gives 18 triples. Add nine triples $A 15, A 19$, $A 59, A 26, A 27, A 67, A 34, A 38, A 48$, and then add nine more triples $B 16, B 18$, $B 68, B 24, B 29, B 49, B 35, B 37, B 57$.

\section{Survey of small bipackings}

With $v=3$, the unique bipacking is (123), (123). With $v=4$, the unique bipacking is the tetrahedral design (123), (124), (134), (234). With $v=5$, the unique bipacking is $A 12, A 13, A 23, B 12, B 13, B 23$. With $v=6$, there is a unique $(6,10,5,3,2)$ design with intersection numbers $x_{1}=6, x_{2}=3$; it has blocks (123), (125), (136), (234), (145), (146), (246), (256), (345), (356).

With $v=7$, there are four non-isomorphic solutions; these are given by putting together two designs $(7,7,3,3,1)$ and may have $0,1,3$, or 7 , repeated blocks; for 
details, see [6]. For $v=8$, we have displayed the twenty-two non-isomorphic solutions in Section 2. For $v=9$, there are 36 non-isomorphic designs $(9,24,8,3,2)$; these appear in the Ph.D. Thesis of E. J. (Morgan) Billington and are also derived in [1]. Another listing without details also appears in [4]. Some of these designs can be found by putting together two affine planes $(9,12,4,3,1)$.

The designs $(10,30,9,3,2)$ for $v=10$ are discussed in [3]; there are many non-isomorphic solutions.

We do not believe that the number of non-isomorphic bipackings has yet been determined for any values of $v$ greater than 10 .

\section{Lambda packings}

In this section, we consider the general question of finding $D_{\lambda}(2,3, v)$; it turns out that the question requires only slight extensions from our knowledge of $D_{1}(2,3, v)$ and $D_{2}(2,3, v)$.

First, we work out the obvious bound on $D_{\lambda}$. By analogy with Lemma 1, we immediately derive

Lemma 4. $D_{\lambda}(1,2, v)=\lfloor\lambda v / 2\rfloor$.

Similarly, we can extend Lemma 3 to

LEMMA 5. $D_{\lambda}(2,3, v) \leqslant\lfloor v\lfloor\lambda(v-1) / 2\rfloor / 3\rfloor$.

We call this bound by the name

$$
\left.B_{\lambda}(2,3, v)=\lfloor v \mid \lambda(v-1) / 2\rfloor / 3\right\rfloor,
$$

and work it out for $v=6 a+b$. The results are given in

THEOREM 11.

$$
\begin{aligned}
B_{\lambda}(2,3,6 a) & =\left(6 a^{2}-2 a\right) \lambda+2 a\lfloor\lambda / 2\rfloor, \\
B_{\lambda}(2,3,6 a+1) & =\lambda\left(6 a^{2}+a\right), \\
B_{\lambda}(2,3,6 a+2) & =\lambda\left(6 a^{2}+2 a\right)+2 a\lfloor\lambda / 2\rfloor+\lfloor 2\lfloor\lambda / 2\rfloor / 3\rfloor, \\
B_{\lambda}(2,3,6 a+3) & =\lambda\left(6 a^{2}+5 a+1\right), \\
B_{\lambda}(2,3,6 a+4) & =\lambda\left(6 a^{2}+6 a+1\right)+(2 a+1)\lfloor\lambda / 2\rfloor+\lfloor(\lambda+\lfloor\lambda / s\rfloor) / 3\rfloor, \\
B_{\lambda}(2,3,6 a+5) & =\left(6 a^{2}-2 a\right) \lambda+2 a\lfloor\lambda / 2\rfloor .
\end{aligned}
$$


Now, if we can achieve a packing in $B_{\lambda}$ blocks, the number of pairs omitted will be

$$
P(\lambda, v)=\lambda v(v-1) / 2-3 B_{\lambda}(2,3, v)
$$

this number is the key to obtaining the packing number $D_{\lambda}(2,3, v)$ and can now be evaluated. The results are given by

THEOREM 12. The number of pairs omitted in a packing that attains the bound $B_{\lambda}(2,3, v)$ is given by:

$$
\begin{aligned}
& P(\lambda, 6 a)=3 a \lambda-6 a\lfloor\lambda / 2\rfloor=0 \quad(\lambda \text { even }) \quad \text { and } 3 a \quad(\lambda \text { odd }) \\
& P(\lambda, 6 a+1)=0 ; \\
& P(\lambda 6 a+2)=\lambda(3 a+1)-6 a\lfloor\lambda / 2\rfloor-3\lfloor 2\lfloor\lambda / 2\rfloor / 3\rfloor ; \\
& P(\lambda, 6 a+3)=0 ; \\
& P(\lambda, 6 a+4)=\lambda(3 a+3)-3(2 a+1)\lfloor\lambda / 2\rfloor-3\lfloor(\lambda+\lfloor\lambda / 2\rfloor) / 3\rfloor \\
& =0 \quad(\lambda \text { even }) \quad \text { and } 3 a+3 \quad(\lambda \text { odd }) ; \\
& P(\lambda, 6 a+5)=\lambda-3\lfloor\lambda / 3\rfloor .
\end{aligned}
$$

Because of the existence of the BIB design with parameter set $(v, v(v-1) t$, $3(v-1) t, 3,6 t)$, it is immediate that, if we set $\lambda=6 t+u$, then these numbers $P(\lambda, u)$ depend only on $u$ and not on $t$. For $v=6 a+b$, we present these numbers in a list involving $u$ and then in a table.

$$
\begin{aligned}
& P(6 t+u, 6 a)=0(u \text { even }) \text { and } 3 a(u \text { odd }) \\
& P(6 t+u, 6 a+1)=0 \\
& P(6 t+u, 6 a+2)=u-3\lfloor u / 3\rfloor(u \text { even }) \text { and } 3 a+u-3\lfloor(u-1) / 3\rfloor(u \\
& \text { dd }) ; \\
& P(6 t+u, 6 a+3)=0 \\
& P(6 t+u, 6 a+4)=0(u \text { even }) \text { and } 3 a+3(u \text { odd }) \\
& P(6 t+u, 6 a+5)=u-3\lfloor u / 3\rfloor .
\end{aligned}
$$
odd);

\begin{tabular}{|c||l|c|c|c|c|c|}
\hline$u$ & 0 & 1 & 2 & 3 & 4 & 5 \\
\hline \hline 0 & 0 & 0 & 0 & 0 & 0 & 0 \\
1 & $3 a$ & 0 & $3 a+1$ & 0 & $3 a+3$ & 1 \\
2 & 0 & 0 & 2 & 0 & 0 & 2 \\
3 & $3 a$ & 0 & $3 a+3$ & 0 & $3 a+3$ & 0 \\
4 & 0 & 0 & 1 & 0 & 0 & 1 \\
5 & $3 a$ & 0 & $3 a+2$ & 0 & $3 a+3$ & 2 \\
\hline
\end{tabular}

TABLE I. Values of $P(6 t+u, 6 a+b)$ 
Now it is impossible for a single pair to be missing from a packing design. So $B_{\lambda}(2,3, v)$ can not be equal to $D_{\lambda}(2,3, v)$ for $v=6 a+2, \lambda=6 t+4$, or for $v=6 a+5, \lambda=6 t+1$ or $\lambda=6 t+4$. In all other cases, the bound is exact.

THEOREM 13. $D_{\lambda}(2,3, v)=B_{\lambda}(2,3, v)$ for all $\lambda$ and $v$ except for $v \equiv 2, \lambda \equiv 4$ $(\bmod 6) ; v \equiv 5, \lambda \equiv 1(\bmod 6) ; v \equiv 5, \lambda \equiv 4(\bmod 6)$. In these three exceptional cases, $D_{\lambda}(2,3, v)=B_{\lambda}(2,3, v)-1$.

Proof. The values of $D_{1}(2,3, v)$ are given in Theorem 4 of [8], and the values of $D_{2}(2,3, v)$ are given in Section 4 .

When $P(\lambda, v)=0$, the bound is attained, since all BIB designs made up of triples exist.

For $v \equiv 0(\bmod 6)$, a BIB design exists for $\lambda$ even. A design omitting $3 a$ pairs exists for $u=1$. Consequently, for $\lambda$ odd, we can achieve the bound by juxtaposing a packing design for $\lambda=1$ and a BIB design for $\lambda-1$.

The same construction of juxtaposing a packing design for $\lambda=1$ and a BIB design for $\lambda-1$ gives a design meeting the bound for $v \equiv 4(\bmod 6)$.

For $v \equiv 5(\bmod 6), \lambda \equiv 1(\bmod 6)$, the bound fails by $1[8]$, and is achieved for $\lambda \equiv 2(\bmod 6)$ [see Section 4$]$. For $\lambda \equiv 4(\bmod 6)$, we can achieve the value $B_{\lambda}(2,3, v)-1$ by using a BIB design for $\lambda-1$ together with a packing design for $\lambda=1$. For $\lambda \equiv 5(\bmod 6)$, we can achieve the bound by using a BIB design for $\lambda-2$ together with a bipacking.

Finally, for $v \equiv 2(\bmod 6)$, we have a packing meeting the bound for $\lambda \equiv 1$ $(\bmod 6)[8]$; we also have a packing for $\lambda \equiv 2(\bmod 6)$ in Section 4 . For $\lambda \equiv 3$ $(\bmod 6)$, we use a packing for $\lambda-2$ together with a bipacking for $\lambda=2$. For $\lambda \equiv 5(\bmod 6)$, take a packing for $\lambda-4$ that omits $(1,2),(3,4), \ldots,(v-1, v)$; and take two bipackings, one that omits pairs $(1,3),(1,3)$, and one that omits pairs $(2,3),(2,3)$. Altogether, we now omit $3 a+5$ pairs and this can be decreased to $3 a+2$ by including the triple 123 . Hence, the only outstanding case is $v \equiv 2$ $(\bmod 6), \lambda \equiv 4(\bmod 6)$.

It will obviously suffice to discuss the case $\lambda=4$, since we can always add arbitrary multiples of the design $(v, v(v-1), 3(v-1), 3,6)$. First, we note that a design with $\lambda=1$ omits $v / 2$ pairs forming a complete one-factor of $K_{t}$. So, if we put together four designs with $\lambda=1$, we get a design omitting four one-factors. For $v=8$, let these one-factors be:

$$
\begin{aligned}
& \text { (12) (34) (56) (78); } \\
& \text { (17) (35) (46) (28); } \\
& \text { (13) (24) (57) (68); } \\
& \text { (14) (26) (58) (37). }
\end{aligned}
$$


Then we can add triples (134), (246), (568), (357), so that only pairs (12), (28), (87), (71), are omitted. With four omitted pairs, we thus achieve the bound $B_{\lambda}(2,3,8)-1$.

This is the beginning of an induction. We have shown that, for $a=1$, we can select four one-factors of $K_{6 a+2}$ so that these one-factors can be grouped into triples and leave only four pairs over. If we suppose this has been done for a particular $v \equiv 2(\bmod 6)$, then we show how to extend the selection to $v+6$. Merely add six new points $A, B, C, D, E, F$, to $K_{v}$ and extend the existing four one-factors by the one-factors

$$
\begin{aligned}
& A C, B D, E F ; A B, C F, D E \\
& A D, B D, C E ; A E, B C, D F
\end{aligned}
$$

This gives us four one-factors of $K_{v+6}$, and we can remove the triples formed from $K_{v}$ together with $A B C, A D E, B D F, C E F$, to leave four pairs, as required (indeed, since these pairs will remain as in $K_{8}$, they will still form the cycle (1287)).

This construction completes the proof of the theorem and the determination of $D_{\lambda}(2,3, v)$.

\section{References}

[1] E. J. (Morgan) Billington, 'Some small quasi-multiple designs', Ars Combinatoria 3 (1977), 233-250.

[2] M. K. Fort and G. A. Hedlund, 'Minimal coverings of pairs by triples', Pacific J. Math. 8 (1958), 709-719.

[3] B. Ganter, R. Mathon and A. Rosa, 'A complete census of $(10,3,2)$-block designs and of Mendelsohn triple systems of order 10', Congressus Numerantium 20 (1977), 383-398.

[4] R. Mathon and A. Rosa, 'A census of Mendelsohn triple systems of order 9', Ars Combinatoria 4 (1977), 309-315.

[5] R. G. Stanton, J. A. Bate and R. C. Mullin, 'Isomorphism classes of a set of prime BIBD parameters', Ars Combinatoria 2 (1976), 251-264.

[6] R. G. Stanton and R. J. Collens, 'A computer system for research on the family classification of BI BD's', Proc. International Congress on Combinatorial Theory, pp. 133-169 (Acad. dei Lincei, Rome 1973).

[7] R. G. Stanton and I. P. Goulden, 'Graph factorization, general triple systems, and cyclic triple systems', Aequationes Math. 22 (1981), 1-28.

[8] R. G. Stanton and J. G. Kalbfleisch, 'Maximal and minimal coverings of $(k-1)$-tuples by $k$-tuples', Pacific J. Math. 26 (1968). 131-140.

[9] A. P. Street, 'Some designs with block size three', Combinatorial Mathematics VII, pp. 224-237 (Lecture Notes in Mathematics 829, Springer-Verlag, 1979). 
[10] R. M. Wilson and D. K. Ray-Chaudhuri, 'Solution of Kirkman's schoolgirl problem', Proc. Symposia Pure Math. 19 (1971), 187-203.

Department of Computer Science

University of Manitoba

Winnipeg, Canada

R3T 2N2
Department of Computer Science

University of Waterloo Waterloo, Canada

N2L 3G1 